\title{
Quantitative Measurement and Comparison of Effects of Various Search Engine Optimization Parameters on Alexa Traffic Rank
}

\author{
Arjun Thakur \\ NIT Jalandhar \\ Jalandhar-144011, India
}

\author{
A. L. Sangal \\ NIT Jalandhar \\ Jalandhar-144011, India
}

\author{
Harminder Bindra \\ NIT Jalandhar \\ Jalandhar-144011, India
}

\begin{abstract}
There are currently 2 billion users on the internet which is roughly $28 \%$ of the global human population. A global market of 2 billion is usually a significant motivator for companies to seriously consider internet branding and marketing. According to Pew Research, people are relying more and more on Internet search. In fact, approximately 50 percent of web users use search every day and search is second only to e-mail in daily tasks performed by web users. These search page views span everything from shopping, travel, entertainment, research, work, local, socializing, sharing, and more. Consequently search engines are the main sources of traffic. More traffic to a commercial website means more visitors, more customers more sales and so high profit in business. It is desirable to bring as much as possible traffic to a company's website. One should consider using SEO (Search Engine Optimization) to increase the website traffic. Alexa Traffic Rank is the most popular website traffic measurement unit. The paper applies various SEO parameters onto a commercial website http://www.ujjaincity.com/ and measures the change in ATR (Alexa Traffic Rank). The paper is intended to help the webmasters and marketing department of a company in improving the ATR of the company's website. Also the paper suggests the relative effectiveness of the different SEO practices so that it can be decided which practice is feasible as well as profitable. With the help of the paper the webmasters and site owners can determine which practices provide good ROI (Return On Investment). The paper will guide them to adopt and implement the best practices to improve the ATR of their website. The improved ATR will lead to more profit in online business.
\end{abstract}

\section{General Terms}

Internet Marketing and Search Engine Optimization.

\section{Keywords}

Alexa Traffic Rank; Commercial Website; Internet Marketing; Search Engine Optimization; Social Media Marketing.

\section{INTRODUCTION}

With the development of E-Commerce, the Internet Marketing becomes more and more important in the new competitive environment $[1,2]$. As Internet becomes ubiquitous, it has been widely noted that technological innovations such as the Internet are a primary driver of industrial productivity. It moves firms beyond the physical constraints of their traditional realm and creates a virtual businesses community [5]. There are currently 2 billion users on the internet which is roughly $28 \%$ of the global human population. A global market of 2 billion is usually a significant motivator for companies to seriously consider internet branding and marketing [3].

A typical local company will invest and develop a high-end website with all the latest technology and functionality. It is launched with much fun-fair and expectation; but soon underperforms or does not deliver on the expectations [3].

Search engine marketing (SEM) refers to improving visibility of a website using different search engine optimization techniques and paid advertisings offered by search engines [1]. Popular search engines, such as Google and Yahoo, have become the interfaces between users and various websites on the Internet. It was estimated that search engines are responsible for nearly $90 \%$ of traffic to most websites [2].

SEM can be of two types paid or non-paid. The paid category includes Paid Placement, Contextual Targeted Text Ads, and Paid Inclusion. The non-paid category includes Organic (Natural) SEO (Search Engine Optimization) [2].

Paid placement is operated by search engines in the form of sponsored or paid results, where an advertisement is displayed in a pre-specified region of a search result page along with web search results. Search engines charge placement fees tied to the price of the relevant keywords, which is primarily determined by auction and measured by CPC (cost per click), and the number of click-throughs the advertisement receives [9].

SEO is the practice of optimizing web pages in a way that improves their ranking in the web search results, which are also known as natural or organic results because they are supposed to reflect relevancy in searchers' standard [9].

SEO is gaining momentum primarily for two reasons. First, $\mathrm{CPC}$ has increased tremendously over years. According to a Fathom Online report, keyword cost has risen $19 \%$ in one year since September 2004[8]. Second, it has been realized that organic results are more appealing to searchers because these results are considered more objective and unbiased than sponsored results.

According to an online survey by Georgia Tech University [10], over $70 \%$ of the search engine users prefer clicking organic results to sponsored results. The SEMPO survey [17] concurs with this finding, showing that organic listings are chosen first by $70 \%$ of the people viewing search results, while sponsored listings receive about $24.6 \%$ of clicks [9]. 
Organic search results might be slow in listing the websites, but its listing is a much more stable and long term process. The search listing which appears through organic search is a more permanent one than PPC which generally runs for a specified time span after which it dissolves and disappears [6].

One should consider using SEO to increase the website traffic. Alexa Traffic Rank is the most popular website traffic measurement unit.

Alexa ranking focuses on website traffic rather than incoming links and therefore, the Alexa Rank is very relevant for sites that get massive targeted traffic. If making money is the goal of the website, then Alexa ranking is the ideal strategy. The lower the Alexa numerical ranking the better for the website. A lower Alexa rank is a clear indication of the website's massive traffic. Alexa Rank also allows webmasters and advertisers get an accurate assessment of our site's ability to generate income. As a result, a better Alexa rank can bring a higher bid for advertising on our site. Since Alexa rank is a snapshot of our website, it is worthwhile to use it as an SEO tool [18].

In 2010, Mo Yunfeng [21] analyzed the impact of receiving and recording of search engines and ranking rules to get understanding of the features of search engine optimization practices commonly used. Yunfeng proposes the optimization tactics for the development of a website for getting high traffic. He proposed tactics for content, for keywords and for links, which are effective for search engine optimization. He estimated the quantitative effect of six SEO affecting factors to guide the industry experts and webmasters. After doing the quantitative measurement he assigned numeric points to each of the factors. We therefore decided to extend the work.

The paper applies various SEO parameters onto a commercial website http://www.ujjaincity.com/ and measures the change in ATR (Alexa Traffic Rank). The paper is intended to help the webmasters and marketing department of a company in improving the ATR of the company's website. Also the paper suggests the relative effectiveness of the different SEO practices so that it can be decided which practice is feasible as well as profitable. With the help of the paper the webmasters and site owners can determine which practices provide good ROI (Return On Investment).

We in this paper apply various parameters on the website and study their comparative effect which will help the webmasters and other stakeholders to put in appropriate and reasonable efforts on various parameters.

The paper is organized as follows. In the section-II we give brief description of search engine architecture and background information about SEO. In section-III there is a brief overview of the website on which we have done our research work. In section-IV we describe various online tools and technologies used in the research work. In section- $\mathrm{V}$ we have given the details of implementation and results. In Section-VI there is a comparison of relative effects of all the parameters. Section-VII concludes the paper followed by the list of references.

\section{SEARCH ENGINE BACKGROUND DEFINITIONS}

AND

\subsection{Search Engine}

Search engine is an information retrieval tool that uses a query of keywords to locate relevant websites on the Internet. Figure 1 shows different components of the search engine architecture and how the components collaborate together to deliver search results (fig. 2) [2].

1) Crawler module - "spiders" or "robots" that constantly search the Internet to collect and categorize information about web pages. The results are stored in a central repository.

2) Page repository - Storage place for complete web pages collected by "spiders" or "robots". Web pages are later sent to the indexing module for processing.

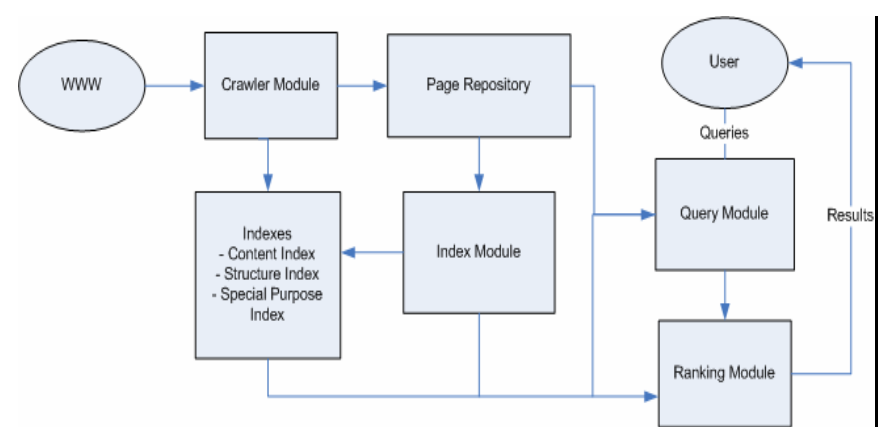

Fig. 1. Search Engine Architecture.

3) Indexing module - Processing unit that extracts vital information from web pages and stores the information in various indexes.

4) Indexes - Storage of the vital information of each web page.

- Content index - Index based on keyword, title, and anchor

text.

- Structured index - Index based on hyperlink structure.

- Special purpose indexes - Index based images and PDF

documents

5) Query module - Processing unit that converts user's natural language query to search query. The query module uses various indexes to return a set of most relevant web pages.

6) Ranking module - Process the set of relevant web pages returned from Query module, and sort them in an order based on the sum of content score and popularity score (overall score) of the web page [2].

\subsection{Keyword}

A single word that relates to a specific subject or topic is called a keyword. Keyword is a specific word or combination of words that a searcher might type into a search field. Includes generic, category keywords; industry-specific terms; product brands; common misspellings and expanded variations all might be entered as a search query. For example, someone looking to buy coffee mugs might use the keyword phrase "ceramic coffee mugs." Also, keywords - which trigger ad network and contextual network ad serves - are the auction components on which PPC advertisers bid for all Ad Groups/Orders and campaigns [37]. 


\section{WEBSITE OVERVIEW}

The website http://www.ujjaincity.com is an information portal of city of Ujjain, the administrative centre of Ujjain District and Ujjain Division India. The site presents information about temple in Ujjain, city news, local business, tourism etc. The website was developed using WordPress technology.

Various SEO parameters were applied onto a commercial website http://www.ujjaincity.com/ and measures the change in ATR.

\section{TOOLS AND TECHNOLOGIES USED}

Right from the website theme selection to an optimized site we have used various tools. A brief description of some of the tools is as follows:

\subsection{Google AdWords}

The Google AdWords is a keyword suggestion tool. This tool will generate a list of similar keywords related to a specific keyword entered. The resulting list is ordered by the keyword's popularity on how often it appears in Google searches. This tool is useful to choosing most effective keywords to advertise or optimize [2]. The tool is available for free: https://adwords.google.com/select/KeywordToolExternal.

\subsection{Google Analytics}

The GA (Google Analytics) is the enterprise-class web analytics solution that gives we rich insights into our website traffic and marketing effectiveness. Powerful, flexible and easy-to-use features let us see and analyze our traffic. With Google Analytics, we record average time on site, sources of traffic etc.

\subsection{Check Domain Name Avaialbility}

To check the availability of a particular domain name or extension there are several tools available online we have used http://www.name.com/. The site was founded in 2003, by Bill Mushkin, who previously founded the computer software company, Mushkin Inc. in 1994 [36].

We used this tools due to it's high speed and ease of use. When we enter a keyword or set of keywords the tool comes up with available domain names and extensions.

\subsection{Alexa Traffic Rank}

Alexa Traffic Rank is obtained by using Alexa tool bar which is available online at http://www.alexa.com/toolbar. The reasons for adopting ATR (Alexa Traffic Rank) as a matrix are as follows:

a. Websites that have a low Alexa rank are good prospects for investments, funding and resale.

b. When a website improves Alexa rank, it develops a better image. If we have an e-commerce website that has poor Alexa rank, it would lose credibility with website visitors and negatively affect sales.

c. A great Alexa rank means more advertising revenue, especially for websites that experience lot of traffic since website ads can result in huge revenue. The lower a website's Alexa rank, the higher its ad revenue.

d. Better website image and credibility when they boost Alexa rank

If our website sees a great amount of traffic, we stand to earn more through advertising since traffic determines Alexa ranking [18].

\subsection{Alexa Traffic Rank Toolbar}

Alexa ranks sites based on tracking information of users of its Alexa Toolbar for Internet Explorer and from integrated sidebars in Firefox and Chrome [14]. When we submit our site to any directories or review sites, the benchmarks which the administrators/editors consider are Google Page Rank and Alexa Traffic Rank [19].

\section{IMPLEMENTATION AND RESULTS}

We considered five parameters for study of variation in ATR. We observed the effect of each of the parameters for seven consecutive days. Apart from this we have not recorded any result in the next five days of the last date of rank recording. This is done so in order to make sure that the effects of two successive parameters do not overlap. We have compared the percentage effect of each parameter to suggest the relative effectiveness of the different SEO practices so that it can be decided which practice is feasible as well as profitable. With the help of the paper the webmasters and site owners can determine which practices provide good ROI from the website optimization and promotion. The following five SEO parameters were applied to the website.

(1)Use of Keywords in the Title of Each Page

(2)Adding ALT Tag to Describe the Images

(3)Social Media Marketing

(4)Adding New Pages Regularly

(5)Correcting Mark-Up Errors

\subsection{Use of Keywords in the Title of Each Page \\ 5.1.1 Introduction}

The <title> tag defines the title of the document. The title element is required in all HTML/XHTML documents. The title element:

a. Defines a title in the browser toolbar.

b. Displays a title for the page in search-engine results

c. Provides a title for the page when it is added to favorites [35].

\subsubsection{Significance}

A title tag tells both users and search engines what the topic of a particular page is. The $\langle$ title $>$ tag should be placed within the $<$ head $>$ tag of the HTML document.

Let us create a unique title for each page on the site.

If the document appears in a search results page, the contents of the title tag will usually appear in the first line of the results (see the SERP snapshot).This can help users recognize if the page is likely to be relevant to their search.

The title for the homepage can list the name of the website / business and could include other bits of important information like the physical location of the business or maybe a few of its main focuses or offerings.

\subsubsection{Implementation of the Parameter (code snippet demo)}

Let us apply the following practices to a page of the site.

a. Use brief, but descriptive titles

b. Accurately describe the page's content

c. Create unique title tags for each page 


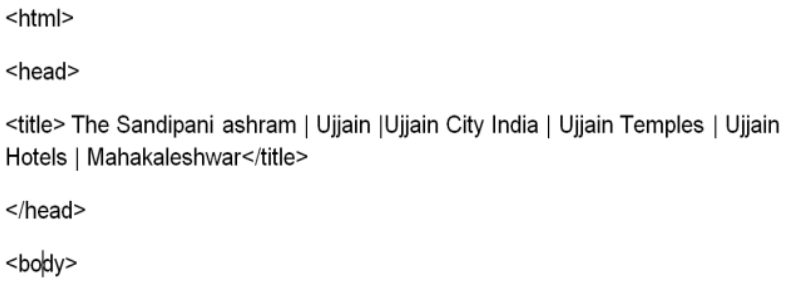

Fig. 2. The title of an inner page for our site, which lists the Sandipani ashram and other main focus areas.

\subsubsection{Querying a Search Engine}

When a search engine is queried the title is highlighted. Refer fig. 3 and fig. 4.

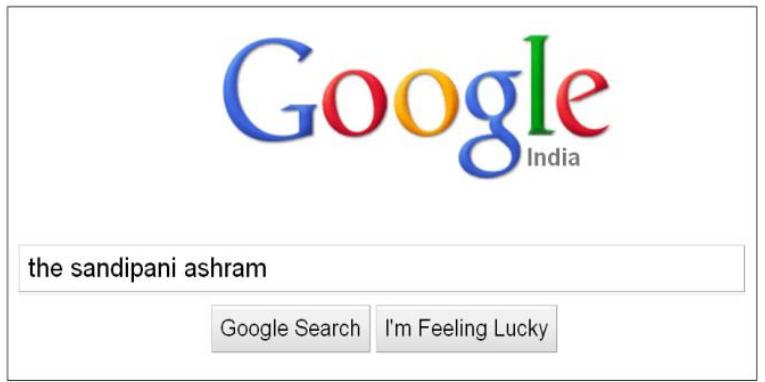

Fig. 3. A user performs the query [the sandipani ashram].

The Sandipani ashram | Ujjain |Ujjain City
India / Ujjain Temples ... Q
The Sandip ... The Sandipani ashram was the first
halt on our temple tour of Ujjain, and we entered the
grounds expecting a short 10 minute halt, ...
city/the-sandipani-ashram/ - Cached

Fig. 4. Our inner page shows up as a result, with the title listed on the first line (notice that the query terms the user searched for appear in bold).

\subsubsection{Results}

The change in ATR of the website from 3 February 2011 to 9 February 2011 is shown in the fig. 5. During the interval the ATR drops from 5686992 to 4933935 , consequently the rank improves by 753057 positions. Thus the effect of the parameter on ATR is of high significance.

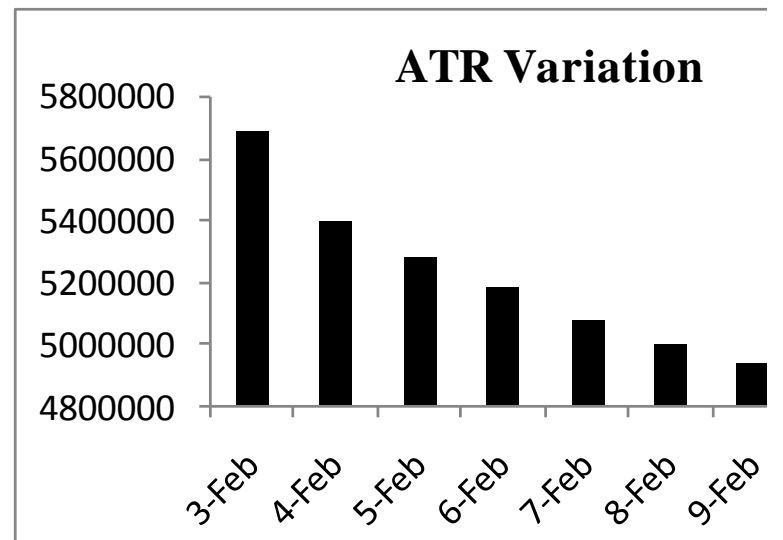

Fig. 5. Variation in ATR of the site after use of keywords in the title of each page.

\subsection{A dding ALT Tag to Describe the Images}

\subsubsection{Introduction}

Images may seem like a straightforward component of the site, but we can optimize the use of them. If a user is viewing our site on a browser that doesn't support images, or is using alternative technologies, such as a screen reader [11], than see will be unable to view the image.

\subsubsection{Significance}

Use the alt attribute to provide descriptive text. In addition, we recommend using a human-readable caption and descriptive text around the image, because search engines have the same problem as blind users. They cannot see the mages.

1. The words used within an image's alt attribute should be its text equivalent and convey the same information or serve the same purpose that the image would [24].

2. The goal is to provide the same functional information that a visual user would see. The alt attribute text should function as a "stand in" in the event that the image itself is not available [24].

\subsubsection{How to Add the ALT tag (code snippet demo)}

Here is the standard format of alt tag of image and is applied to the website www.ujjaincity.com.

<img src="http://www.ujiaincity.com/wpcontent/uploads/2011/03/Gopal-Mandir-Ujiain-225x300.jpg" alt="Gopal Mandir Ujjain, Krishna temples in Ujjain" />

Fig. 6. The ALT tag is added to each image of the site one of the example is shown in the figure. 


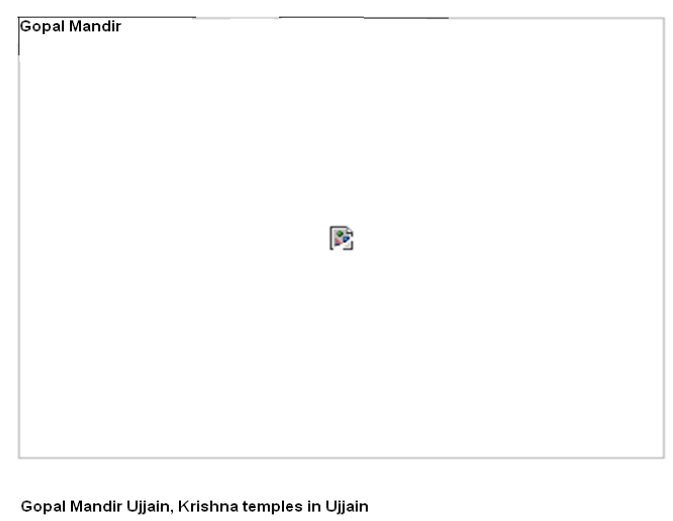

Fig. 7. The image wasn't displayed to the user for some reason, but at least the alt text was.

Adding the ALT tag is particularly useful when the browser does not support the image format or the user is browsing the web with mobile phone that has problem loading or displaying images.

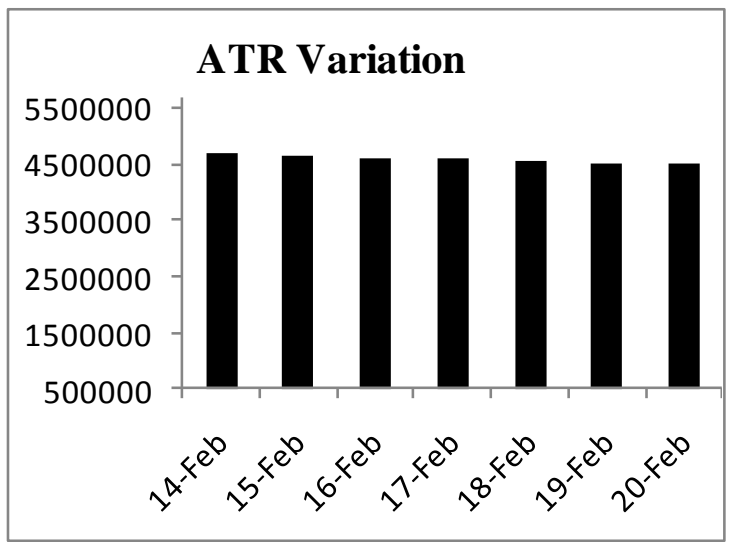

Fig. 8. Variation in ATR of the site after use of ALT tag for each image of the website.

\subsubsection{Results}

The change in ATR of the website from 14 February 2011 to 20 February 2011 is shown in the fig. 8. During the interval the ATR drops from 4708019 to 4489289 , consequently the rank improves by 218729 positions. Thus the effect of the parameter on ATR is of moderate significance.

\subsection{Social Media Marketing}

\subsubsection{Inroduction}

Social media marketing is a recent addition to organizations' integrated marketing communications plans. Integrated marketing communications is a practice organizations follow to connect with their target markets. Integrated marketing communications coordinates promotional elements: advertising, personal selling, public relations, publicity, direct marketing and sales promotion [32].

Social media marketing programs usually center on efforts to create content that attracts attention and encourages readers to share it with their social networks. A corporate message spreads from user to user and presumably resonates because it is coming from a trusted, third-party source, as opposed to the brand or company itself [31].

Social media has become a platform that is easily accessible to anyone with internet access. Increased communication for organizations fosters brand awareness and often, improved customer service. Additionally, social media serves as a relatively inexpensive platform for organizations to implement marketing campaigns. With emergence of channels like Twitter, the barrier to entry in social media is greatly reduced [31].

\subsubsection{Significance}

The 2008 presidential campaign had a huge presence on social networking sites. Democratic candidate, Barack Obama, was very visible on Twitter and Facebook .His social networking site profile pages were constantly being updated and interacting with followers. The use of social networking sites gave Barack Obama's campaign access to e-mail addresses, as posted on social networking site profile pages. This allowed the Democratic Party to launch e-mail campaigns asking for votes and campaign donations [31].

\subsubsection{How to Do Social Media Marketing}

Here To implement the above strategies on WordPress site we need to install the plugin on the page http://www.ujjaincity.com/wp-admin/plugin-install.phpThis plugin adds a footer link to add the current post or page as a Facebook link. While the plugin is activated a link will appear after the content of each post/page with the text "Share on Facebook", the Facebook icon, both or the familiar Facebook share button. Clicking the link will bring the user to the Facebook site to add the link to their profile. If the user isn't logged in they will be prompted to do so [33].

Similarly we can perform social media marketing on Twitter, LinkedIn, YouTube etc. Consequently we get social media marketing buttons on each page of our website (see fig. 9.)

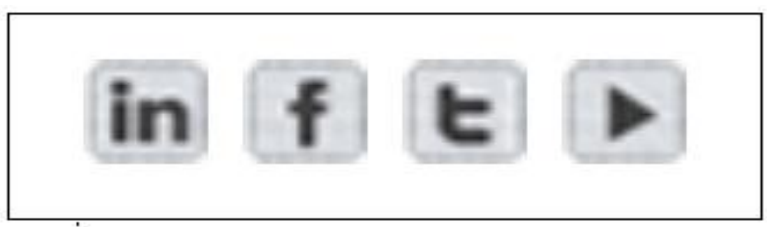

Fig. 9. Social Media Marketing Buttons for LinkedIn, Facebook, Twitter, YouTube respectively.

\subsubsection{Results}

The change in ATR of the website from 25 February 2011 to 3 March 2011 is shown in the fig. 10. During the interval the ATR drops from 4428044 to 4096825 , consequently the rank improves by 331219 positions. Thus the effect of the parameter on ATR is of moderate significance. 


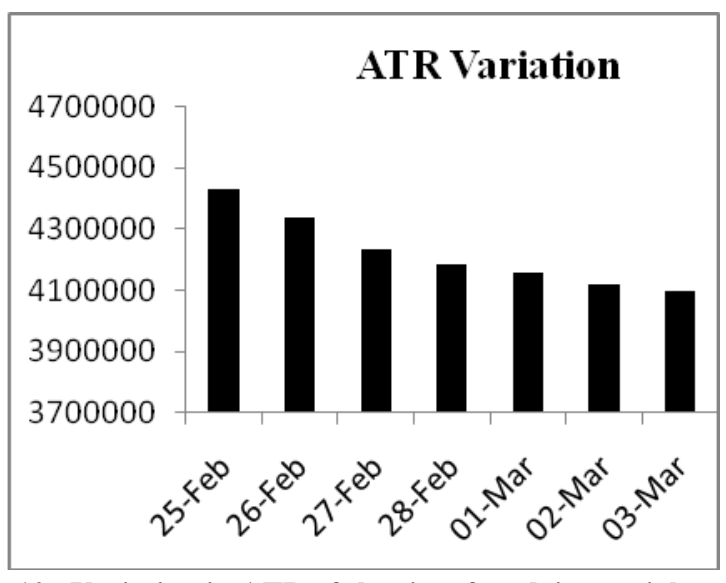

Fig. 10. Variation in ATR of the site after doing social media marketing

\subsection{Adding New Pages Regularly}

\subsubsection{Introduction}

Adding new pages is important since this is how a site can offer a visitor more information. When a new page is added to a website it will be crawled by a search engine spider. The page that is crawled will also be indexed, consequently it will show up in SERPs of various search engines.

\subsubsection{Significance}

As a new page is added to a website the content will be available to the user hence it brings more and more traffic to the website.

\subsubsection{How to apply the parameter}

It was rather difficult to measure the quantitative effect of this parameter. From the date 8 March 2011 to 14 March 2011 we have added pages to the site regularly. The change in ATR is shown in the result graph.

\subsubsection{Results}

The change in ATR of the website from 8 March 2011 to 14 March 2011 is shown in the fig. 11. During the interval the ATR drops from 3990897 to 3419074 , consequently the rank improves by 571822 positions. Thus the effect of the parameter on ATR is of high significance.

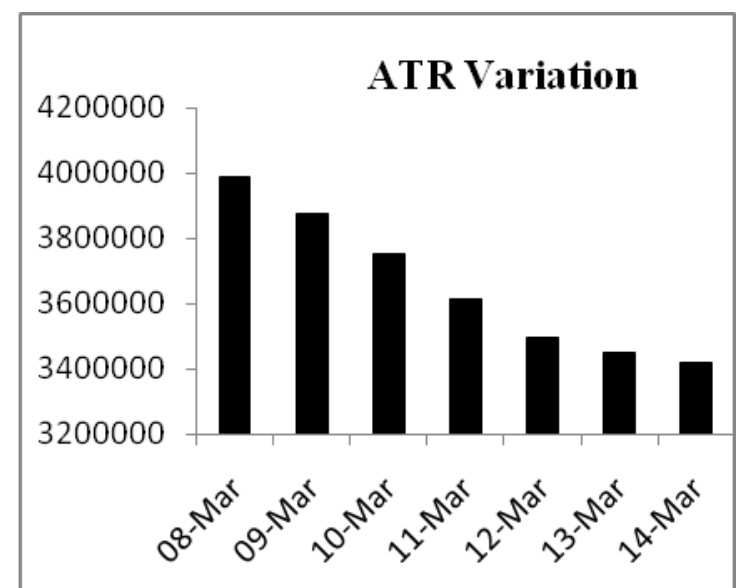

Fig. 11. Variation in ATR of the site after regularly uploading new contents to the website.

\subsection{Correcting Mark-Up Errors}

\subsubsection{Introduction}

The Markup Validation Service is a validator by the World Wide Web Consortium (W3C) that allows internet users to check HTML documents for conformance to HTML or XHTML standards. It also provides a quick method for web page authors to check their posted pages for mark-up errors [30].

\subsubsection{How to Correct Mark-Up Errors}

The validation can be carried out using the online tool available at http://validator.w3.org/. All we have to do is to enter the homepage url and the tool checks and validates the markup.HTML validators operate by comparing the mark-up on a web page to the $\mathrm{W} 3 \mathrm{C}$ standards. The standards vary depending upon the declared version and so the validator will start by reading the DOCTYPE declaration to see which set of standards to apply. Once the validator has read the page and determined the applicable standards it looks for such things as missing opening or closing tags, missing quotation marks and other hand-coding errors. The validator then provides a report indicating that the coding is correct or not. Errors are noted in a list. One error, such as neglecting to close a tag, can cause a cascade of errors through the page, producing dozens or even hundreds of noted errors [30].

\subsubsection{Results}

The change in ATR of the website from 19 March 2011 to 25 March 2011 is shown in the fig. 12. During the interval the ATR drops from 3247527 to 3141287 , consequently the rank improves by 106240 positions. Thus the effect of the parameter on ATR is of low significance. 


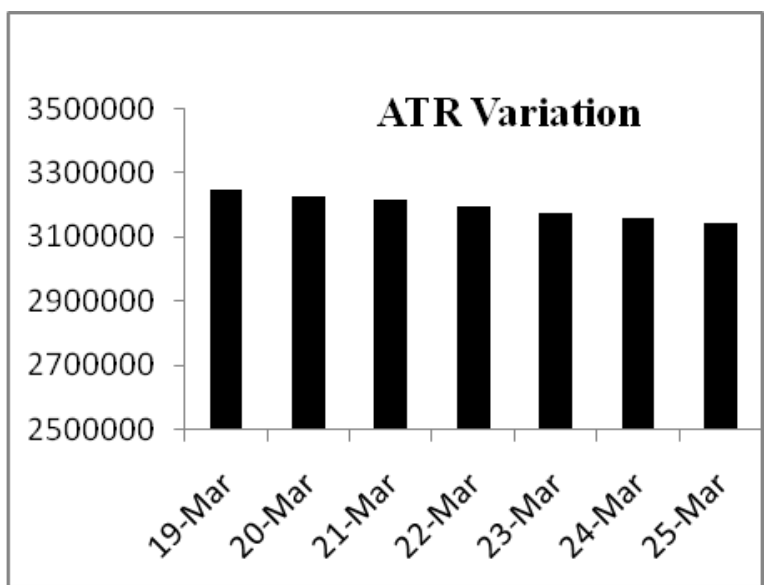

Fig. 12. Variation in the ATR of the website after correcting markup errors.

\section{COMPARISON OF ALL THE RESULTS}

After studying the effect of each individual parameter we study the pie-chart to compare the percent effects of the parameters refer to fig. 13 .

Results indicate that the percent effect of this parameter "use of keywords in the title of each page" is $38 \%$. This is of high importance; at the same time it can easily be implemented using Google AdWords tool. We therefore highly recommended implement this parameter; it can easily be done by following the practice as explained in implementation and results part of the paper.

Pie-chart results indicate that the percent effect of adding alt tag to images is $11 \%$. Consequently the parameter has a moderate impact on overall ATR improvement. In section 5.4 of the chapter we described how to implement this parameter. It is relatively straightforward to apply this on a WordPress site. We hereby recommend to apply these practices to a website for good ROI.

Pie-chart results indicate that the percent effect of social media marketing is $17 \%$. Consequently the parameter has a moderate impact on overall ATR improvement. in implementation and results part of the paper, we described how to implement this parameter. It is relatively straightforward to apply this on a WordPress site. We hereby recommend to apply these practices to a website for good ROI.

Pie-chart results indicate that the percent effect of adding new pages regularly this $29 \%$. Consequently the parameter has a high impact on overall ATR improvement. in implementation and results part of the paper, we described how to implement this parameter. It is a slow process to apply this. However it will be good for the visitors, hence we highly recommend to apply these practices to a website for good ROI.

Pie-chart results indicate that the percent effect of this parameter is only $3 \%$. Consequently the parameter has a very low impact on overall ATR improvement. in implementation and results part of the paper, we described how to implement this parameter. It is relatively tedious to apply this on a WordPress site. We hereby NOT recommend to apply these practices to a website for good ROI.

\section{The Pie-Chart of ATR Affecting Factors}

Use of Keywords in the Title of Each Page

- Adding ALT Tag to Describe the Images of the Website

Social Media Marketing

- Adding New Pages Regularly

Correcting Mark-Up Errors

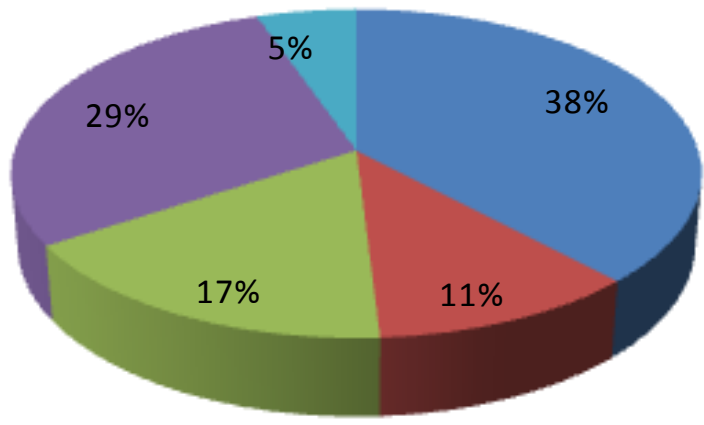

Fig. 13. The Pie-Chart of ATR Affecting Factors

\section{CONCLUSION}

The research on variation in ATR due to SEO factors has great significance on academic theory and broad application prospects. Its application leaves more room for improvement waiting for

scholars' in-depth study and exploration.

With the help of this paper the website owners and webmasters can decide which SEO practice is worth and will give a good return on investment. The paper will guide them to adopt and implement the best practices to improve the ATR of their website. The improved ATR will lead to more profit in online business.

The present paper proposes the impact of keyword in title tag, ALT tag for images, social media marketing, uploading new contents regularly and markup validation. The work can be extended by studying the impact of meta-tags, average time on site, use of xml sitemaps, use of advertisement on the web pages etc.

Nevertheless, along with the development of search engine technologies, we need to continuously find new tactics for securing good ATR which is a subject of long-term and constant study. 


\section{REFERENCES}

[1] Thurow S, "Search Engine Visibility, Berkeley", New Riders Press, 2003

[2] Weider D. Yu, Andy Lin, "The Design and Implementation of A Search Engine Marketing Management System (SEMMS) Based on Service-Oriented Architecture Platform" IEEE International Conference on e-Business Engineering 0-7695-3003-6/07 \$25.00 (C) 2007 IEEE DOI 10.1109/ICEBE.2007.117, page 9513-5190, retrieved July 3, 2006,

[3] Daily Mirror, "Internet Marketing" http://print.dailymirror.lk/business/127-local/38977.html, last accessed on May 5, 2011

[4] SEMPO, "The State of Search Engine Marketing, 2005" from http://www.sempo.org/learning_center/research/ , last accessed on July 3, 2006

[5] Meng Jiajia, Dong Dahai, "Determinants and Effect of Internet Marketing in E-Commerce: An Empirical Investigation from China" 2010 International Conference on E-Business and E-Government , 978-0-7695-3997-3/10 (C) 2010 IEEE DOI 10.1109/ICEE.2010.75, page 267-270, 2010

[6] Selvaseo, "Organic SEO" http://www.selvaseo.com/organic-seo-for-long-termbenefits/ last accessed on May 7, 2011

[7] OYLINKI, "the benefits of organic SEO" http://oylinki.com/2009/12/30/the-benefits-of-organic-seosearch-engine-optimization-3.html last accessed on May 7, 2011.

[8] WordPress, "Natural SEO", http://micrositez10.wordpress.com/2011/03/15/advantageof-natural-seo-over-paid-seo/ last accessed on May 7, 2011.

[9] Bo Xing, Zhangxi Lin, "The Impact of Search Engine Optimization on Online Advertising Market" Conference'04, Month 1-2, 2004, Copyright 2004, ACM 1-58113-000-0/00/0004, page 519-529, May 7, 2011

[10] DataSprings,

SEO, http://www.datasprings.com/resources/articlesinformation/seo-search-engine-optimization last accessed on May 7, 2011

[11] Google, SEO Guide, http://www.google.com/webmasters/docs/search-engineoptimization-starter-guide.pdf last accessed on May 7, 2011

[12] Bing, New Features Relevant to Webmasters June 1, 2009 "Bing Webmaster Center Team," Microsoft ${ }^{\circledR}$, June 1, 2009

[13] SEO Articles, spiders, http://www.seoarticles.com/2010/02/11/understanding-theuse-of-seo-spiders/ last accessed on December 1, 2010

[14] Wikipedia, "Alexa Internet" http://en.wikipedia.org/wiki/Alexa_Internet last accessed on May 10, 2011
[15] Alexa, http://www.alexa.com last accessed on May 10, 2011

[16] WordStream Tools, http://www.wordstream.com/adwordskeyword-tool last accessed on May 10, 2011

[17] Savvyk, Tools, http://www.savvyk.com/google-adwordskeyword-tool/ last accessed on May 10, 2011

[18] Alexa Traffic Rank, http://www.youralexaranking.com/alexa-rank-FAQ.html last accessed on May 12, 2011

[19] TeamNirvana Comparison, http://teamnirvana.com/blog/which-is-important-googlepage-rankpr-or-alexa-traffic- rank.html last accessed on May 12, 2011

[20] PageRank, http://webdesignserviceindia.blogspot.com/2009/02/pagerank-advantages- disadvantages.html last accessed on May 15,2011

[21] Mo Yunfeng, "A Study on Tactics for Corporate Website Development Aiming at Search Engine Optimization", 2010 Second International Workshop on Education Technology and Computer Science, 978-0-7695-3987-4/10 $\$ 26.00$ (C) 2010 IEEE DOI 10.1109/ETCS.2010.230, page (673-675) , 2010

[22] Alexa Inc., http://www.alexa.com/help/traffic-learn-more last accessed on may 17, 2011

[23] Alexa Inc., http://www.alexa.com/faqs/?cat=4 last accessed on May 17,2011

[24] SEO Workers, "Alt Tag", http://www.seoworkers.com/seoarticles-tutorials/alt-attribute.html last accessed on May 17, 2011

[25] Wikipedia, "sitemap", http://en.wikipedia.org/wiki/Site_map last accessed on May 18,2011

[26] w3, "Validator", http://validator.w3.org/, last accessed on May 18, 2011

[27] WordPress http://wordpress.org/extend/plugins/google-sitemapgenerator/ , last accessed on May 18, 2011

[28] Oreilly, "ATS", http://toc.oreilly.com/resources/web_average_time_on_site. html last accessed on May 19, 2011

[29] VisualRevenue, Definitions, http://visualrevenue.com/blog/2007/08/avg-time-per-visitstandard-definition.html, last accessed on May 19, 2011

[30] Wikipedia, http://en.wikipedia.org/wiki/W3C_Markup_Validation_Ser vice, last accessed on May 19, 2011

[31] Wikipedia, "SMM", http://en.wikipedia.org/wiki/Social_media_marketing, last accessed on May 19, 2011 
[32] Facebook, http://www.facebook.com/pages/Socialnetwork-marketing/110249788998293 last accessed on May 19, 2011

[33] UjjainCity Admin, http://www.ujjaincity.com/wpadmin/plugininstall.php?tab=search\&type=term\&s=share + on + facebook \&plugin-search-input=Search+Plugins, last accessed on May 19, 2011

[34] WaitPainCentral, "About Contents" http://www.wetpaintcentral.com/page/Create+Unique+Con tent last accessed on May 20, 2011
[35] w3schools, "title tag" http://www.w3schools.com/TAGS/tag_title.asp last accessed on May 20, 2011

[36] Wikipedia, http://en.wikipedia.org/wiki/Name.com last accessed on May 25, 2011

[37] SEMPO, http://www.sempo.org/?page=glossary\#k last accessed on May 26, 2011 